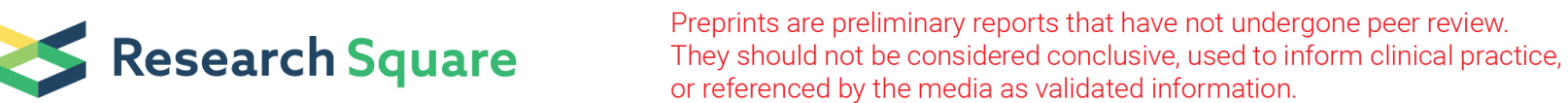

\section{The Association of Metabolic Syndrome with the Initiation of Antidepressive Agents: Real-World Data Analysis of Administrative Claims and Health Examinations}

Takahiro Imaizumi ( $\Delta$ imaizumi18@med.nagoya-u.ac.jp)

Nagoya University Hospital

Takuya Toda

Prevent Co.,Ltd

Michitaka Maekawa

Hidamari kokoro clinic

Daisuke Sakurai

Prevent Co.,Ltd

Yuta Hagiwara

Prevent Co.,Ltd

Yasuko Yoshida

Nagoya University

Masahiko Ando

Nagoya University Hospital

Shoichi Maruyama

Nagoya University Graduate School of Medicine

Research Article

Keywords:

Posted Date: February 10th, 2022

DOI: https://doi.org/10.21203/rs.3.rs-1302206/v1

License: (9) This work is licensed under a Creative Commons Attribution 4.0 International License.

Read Full License 


\section{Abstract}

Depression and metabolic syndrome (MetS) are correlated, leading to an increased healthcare burden and decreased productivity. We aimed to investigate the associations of medical conditions, including MetS and lifestyle, with depression, using a health checkup and claims database. Individuals aged 18-64 years who underwent health examinations between 2014 and 2019 were enrolled in the study. Among 81,269 participants, "ever" and "incident" antidepressant users exhibited worse metabolic profiles and underwent more prevalent cancer-related hospitalization than "never" users. In a nested case-control study with a 1:10 ratio of incident users to controls, MetS (odds ratio, 1.29 [95\% confidence interval: 1.08-1.54]); "quicker" (0.69 [0.59-0.81]) and "late" eating speed (1.25 [1.07-1.46]), as compared with "normal"; poor sleep (1.41 [1.25-1.60]); "sometimes" (0.85 [0.73-0.98]) and "everyday" drink (0.78 [0.66$0.92])$, as compared with "rarely drink"; and hypnotic (1.97 [1.47-2.64]), anxiolytic (3.55 [2.79-4.51]), and nonsteroidal anti-inflammatory drug (1.65 [1.18-2.32]) use were associated with incident antidepressant use. A bodyweight increase $>10 \mathrm{~kg}$ from age 20 years $(1.29$ [1.14-1.46]) was also significantly associated with incident antidepressant use. In conclusion, metabolic abnormalities, hospitalization records, and lifestyle information can be useful in identifying populations at high risk of depression.

\section{Introduction}

Depression is one of the most common mental illnesses affecting working-age adults and is a major public health problem worldwide. ${ }^{1}$ Depression is associated with increased mortality in the general population and among persons with comorbidities. ${ }^{2-5} \mathrm{~A}$ growing body of evidence demonstrates that depression is associated with an increased risk of physical disease, including diabetes mellitus ${ }^{6,7}$ and cardiovascular disease (CVD). ${ }^{2,8-10}$ Furthermore, mental disorders including depression account for approximately $20-50 \%$ of disability benefits across the Organisation for Economic Co-operation and Development countries ${ }^{11}$ and have been found to significantly reduce the ability of people to work by increasing other disabilities, including physical ailments ${ }^{12,13}$. Thus, depression not only has the potential to increase net healthcare costs for the treatment of various physical illnesses in addition to depression, but can also cause presenteeism and absenteeism, which potentially lead to lost productive time in the workplace. ${ }^{14,15}$

Various comorbidities, such as cancer, stroke, and metabolic syndrome (MetS), are associated with the incidence of depression. ${ }^{16-20}$ A systematic review revealed that depression is two to three times more likely to occur in people with multimorbidity than in those without multimorbidity or those with no chronic physical condition. MetS is a pre-symptomatic state and is defined as a combination of abdominal or visceral obesity, hypertension, dyslipidemia, and glucose dysregulation, and it has diagnostic significance in predicting subsequent coronary artery disease, metabolic diseases, and certain cancers. ${ }^{21,22}$ In Japan, universal health screening and education programs were launched in the early 2000s, and their main objective was to detect high-risk populations for CVD. ${ }^{23}$ MetS is strongly associated with lifestyle, especially a sedentary lifestyle and low physical activity, and a relatively high-energy diet potentially leads 
to MetS. Prior studies have shown an association between MetS and depression ${ }^{19,20}$; however, few longitudinal studies have demonstrated this association, and knowledge regarding its association with lifestyle remains limited.

Therefore, this study aimed to examine the association of medical conditions, such as medication and prior hospitalization, and lifestyle with the incident use of antidepressants, with a particular focus on its association with MetS. We used health insurance claims and health checkup data of corporate insurance beneficiaries, who are representative of the major workforce in Japan. This study provides evidence that indicates a relative association of these factors with depression and paves the way for a new approach to preventing depression.

\section{Results}

\section{Baseline characteristics of the overall study population}

Individuals aged 18-64 years who underwent health examinations between April 1, 2014, and March 31, 2019 , were enrolled in the study. The flow diagram shows the study population and overall study design (Figure 1). Participants with the following characteristics were excluded: a lookback period $<365$ days (n $=24,103)$; end-stage kidney disease, defined as prevalent dialysis at baseline or an estimated glomerular filtration rate $<15 \mathrm{~mL} / \mathrm{min} / 1.73 \mathrm{~m}^{2}(\mathrm{n}=48)$; use of second generation antipsychotics or lithium $(n=454)$; missing information on the following metabolic traits: body mass index (BMI), blood pressure (BP), or abdominal circumference (AC); missing laboratory data on hemoglobin A1c (HbA1c), fasting blood glucose, triglycerides (TG), high-density lipoprotein cholesterol (HDL-c), or low-density lipoprotein cholesterol (LDL-C); or missing questionnaire on bodyweight change from age 20 ( $n=28,803$ ). In the descriptive cohort, 81,269 individuals were eligible for the study, including 1,797 individuals who had ever used or were currently using antidepressants, 78,142 who had never used antidepressants, and 1,330 incident users of antidepressants after baseline.

The baseline characteristics of "ever", "never", and "incident" users of antidepressants in the descriptive cohort are shown in Table 1. Ever users had a higher proportion of diabetes mellitus and dyslipidemia cases than the other users. Ever and incident users had a high proportion of MetS cases. Questionnaires on lifestyle revealed that never users were less likely to experience bodyweight increase and poor sleep and more likely to be physically active. Late supper, skipping breakfast, and eating speed were not clinically different across the groups. The questionnaire on bodyweight change showed that ever and incident users were more likely to experience bodyweight increase from the age of 20. Regarding alcoholdrinking habits, never users had a lower proportion of occasional or daily drinkers than the others. 
Table 1

Baseline characteristics of the descriptive cohort

\begin{tabular}{|c|c|c|c|c|c|}
\hline & $\mathbf{N}$ & $\begin{array}{l}\text { Total } \\
(81,269)\end{array}$ & $\begin{array}{l}\text { Never users } \\
(78,142)\end{array}$ & $\begin{array}{l}\text { Incident } \\
\text { users } \\
(1,330)\end{array}$ & $\begin{array}{l}\text { Ever users } \\
(1,797)\end{array}$ \\
\hline Age & 81,269 & $46(9)$ & $46(9)$ & $46(8)$ & $45(8)$ \\
\hline Sex & 81,269 & $\begin{array}{l}48,610 \\
(59.8)\end{array}$ & $\begin{array}{l}46,504 \\
(59.5)\end{array}$ & $886(66.6)$ & $1,220(67.9)$ \\
\hline \multicolumn{6}{|l|}{ Metabolic traits } \\
\hline Metabolic syndrome & 81,269 & $9,019(11.1)$ & $8,543(10.9)$ & $190(14.3)$ & $286(15.9)$ \\
\hline Abdominal circumference & 81,269 & $82(10)$ & $81(10)$ & $83(10)$ & $83(10)$ \\
\hline BMI & 81,269 & $23.0(3.7)$ & $23.0(3.7)$ & $23.5(3.9)$ & $23.7(3.9)$ \\
\hline Systolic blood pressure & 81,269 & $120(16)$ & $120(16)$ & $121(15)$ & $121(15)$ \\
\hline Diastolic blood pressure & 81,269 & $74(11)$ & $74(11)$ & $75(11)$ & $75(11)$ \\
\hline Triglyceride & 81,269 & $\begin{array}{l}83(58- \\
124)\end{array}$ & $\begin{array}{l}82(58- \\
123)\end{array}$ & $\begin{array}{l}91(61- \\
133)^{-}\end{array}$ & $\begin{array}{l}95(65- \\
147)\end{array}$ \\
\hline HDL cholesterol & 81,269 & $62(52-75)$ & $63(52-75)$ & $60(51-71)$ & $60(50-72)$ \\
\hline LDL cholesterol & 81,266 & $\begin{array}{l}119(100- \\
141)\end{array}$ & $\begin{array}{l}119(100- \\
141)\end{array}$ & $\begin{array}{l}122(102- \\
144)\end{array}$ & $\begin{array}{l}123(103- \\
143)\end{array}$ \\
\hline $\mathrm{HbA1c}$ & 63,656 & $\begin{array}{l}5.5(5.3- \\
5.7)\end{array}$ & $\begin{array}{l}5.5(5.3- \\
5.7)\end{array}$ & $\begin{array}{l}5.5(5.3- \\
5.7)\end{array}$ & $\begin{array}{l}5.5(5.3- \\
5.8)\end{array}$ \\
\hline Fasting blood glucose & 81,269 & $\begin{array}{l}93(87- \\
101)\end{array}$ & $\begin{array}{l}93(87- \\
101)\end{array}$ & $\begin{array}{l}95(88- \\
102)\end{array}$ & $\begin{array}{l}95(88- \\
103)\end{array}$ \\
\hline \multicolumn{6}{|c|}{ Self-administered lifestyle information } \\
\hline Exercise $>30 \mathrm{~min} /$ day & 75,543 & $\begin{array}{l}13,739 \\
(18.2)\end{array}$ & $\begin{array}{l}13,260 \\
(18.3)\end{array}$ & $208(16.8)$ & $271(15.9)$ \\
\hline $\begin{array}{l}\text { Physical activity }>60 \\
\mathrm{~min} / \text { day }\end{array}$ & 75,461 & $\begin{array}{l}34,172 \\
(45.3)\end{array}$ & $\begin{array}{l}32,886 \\
(45.4)\end{array}$ & $551(44.5)$ & $735(43.0)$ \\
\hline Fast walking speed & 24,397 & $\begin{array}{l}11,349 \\
(46.5)\end{array}$ & $\begin{array}{l}11,044 \\
(46.7)\end{array}$ & $117(39.3)$ & $188(41.8)$ \\
\hline Eating speed & 75,501 & & & & \\
\hline
\end{tabular}

Data are expressed as $\mathrm{N}(\%)$ for categorical values and mean (standard deviation) for continuous values. Abbreviations: BMI, body mass index; SBP, systolic blood pressure; DBP, diastolic blood pressure; HDL, high-density lipoprotein; LDL, low-density lipoprotein; BW, body weight; IHD, ischemic heart disease; CVD, cardiovascular disease; CHF, congestive heart failure; NSAIDs, non-steroidal antiinflammatory drugs 


\begin{tabular}{|c|c|c|c|c|c|}
\hline & $\mathbf{N}$ & $\begin{array}{l}\text { Total } \\
(81,269)\end{array}$ & $\begin{array}{l}\text { Never users } \\
(78,142)\end{array}$ & $\begin{array}{l}\text { Incident } \\
\text { users } \\
(1,330)\end{array}$ & $\begin{array}{l}\text { Ever users } \\
(1,797)\end{array}$ \\
\hline Quicker & & $\begin{array}{l}15,799 \\
(20.9)\end{array}$ & $\begin{array}{l}15,236 \\
(21.0)\end{array}$ & $231(18.6)$ & $332(19.5)$ \\
\hline Normal & & $\begin{array}{l}45,551 \\
(60.3)\end{array}$ & $\begin{array}{l}43,790 \\
(60.4)\end{array}$ & $756(60.9)$ & $1,005(58.9)$ \\
\hline Late & & $\begin{array}{l}14,151 \\
(18.7)\end{array}$ & $\begin{array}{l}13,528 \\
(18.6)\end{array}$ & $254(20.5)$ & $369(21.6)$ \\
\hline Late supper & 75,478 & $\begin{array}{l}26,675 \\
(35.3)\end{array}$ & $\begin{array}{l}25,490 \\
(35.1)\end{array}$ & $515(41.5)$ & $670(39.2)$ \\
\hline Skipping breakfast & 71,899 & $\begin{array}{l}10,992 \\
(15.3)\end{array}$ & $\begin{array}{l}10,482 \\
(15.2)\end{array}$ & $223(18.7)$ & $287(17.3)$ \\
\hline Poor sleep & 75,455 & $\begin{array}{l}25,533 \\
(33.8)\end{array}$ & $\begin{array}{l}24,260 \\
(33.5)\end{array}$ & $552(44.5)$ & $721(42.3)$ \\
\hline Drinking habits & 80,503 & & & & \\
\hline Rarely drink & & $\begin{array}{l}37,079 \\
(46.1)\end{array}$ & $\begin{array}{l}35,530 \\
(45.9)\end{array}$ & $632(48.0)$ & $917(51.4)$ \\
\hline Sometimes & & $\begin{array}{l}24,600 \\
(30.6)\end{array}$ & $\begin{array}{l}23,721 \\
(30.6)\end{array}$ & $395(30.0)$ & $484(27.1)$ \\
\hline Everyday & & $\begin{array}{l}18,824 \\
(23.4)\end{array}$ & $\begin{array}{l}18,151 \\
(23.5)\end{array}$ & $291(22.1)$ & $382(21.4)$ \\
\hline Current smoking & 81,143 & $\begin{array}{l}18,970 \\
(23.4)\end{array}$ & $\begin{array}{l}18,138 \\
(23.2)\end{array}$ & $351(26.4)$ & $481(26.8)$ \\
\hline $\begin{array}{l}>10 \mathrm{~kg} \text { increase in BW from } \\
\text { age } 20\end{array}$ & 81,269 & $\begin{array}{l}29,874 \\
(36.8)\end{array}$ & $\begin{array}{l}28,448 \\
(36.4)\end{array}$ & $594(44.7)$ & $832(46.3)$ \\
\hline \multicolumn{6}{|c|}{ Current and past medical history } \\
\hline Hypertension & 81,269 & $\begin{array}{l}15,740 \\
(19.4)\end{array}$ & $\begin{array}{l}15,072 \\
(19.3)\end{array}$ & $273(20.5)$ & $395(22.0)$ \\
\hline Diabetes mellitus & 81,269 & $4,722(5.8)$ & $4,498(5.8)$ & $76(5.7)$ & $148(8.2)$ \\
\hline Dyslipidemia & 81,269 & $\begin{array}{l}28,627 \\
(35.2)\end{array}$ & $\begin{array}{l}27,349 \\
(35.0)\end{array}$ & $506(38.0)$ & $772(43.0)$ \\
\hline Self-reported stroke & 80,399 & $326(0.4)$ & $314(0.4)$ & $7(0.5)$ & $5(0.3)$ \\
\hline
\end{tabular}

Data are expressed as $\mathrm{N}(\%)$ for categorical values and mean (standard deviation) for continuous values. Abbreviations: BMI, body mass index; SBP, systolic blood pressure; DBP, diastolic blood pressure; HDL, high-density lipoprotein; LDL, low-density lipoprotein; BW, body weight; IHD, ischemic heart disease; CVD, cardiovascular disease; CHF, congestive heart failure; NSAIDs, non-steroidal antiinflammatory drugs 


\begin{tabular}{|llllll|}
\hline N & $\begin{array}{l}\text { Total } \\
(\mathbf{8 1 , 2 6 9 )}\end{array}$ & $\begin{array}{l}\text { Never users } \\
(\mathbf{7 8 , 1 4 2 )}\end{array}$ & $\begin{array}{l}\text { Incident } \\
\text { users } \\
\mathbf{( 1 , 3 3 0 )}\end{array}$ & $\begin{array}{l}\text { Ever users } \\
\mathbf{( 1 , 7 9 7 )}\end{array}$ \\
\hline Self-reported IHD & 80,409 & $808(1.0)$ & $771(1.0)$ & $12(0.9)$ & $25(1.4)$ \\
\hline $\begin{array}{l}\text { Data are expressed as N (\%) for categorical values and mean (standard deviation) for continuous } \\
\text { values. Abbreviations: BMI, body mass index; SBP, systolic blood pressure; DBP, diastolic blood } \\
\text { pressure; HDL, high-density lipoprotein; LDL, low-density lipoprotein; BW, body weight; IHD, ischemic } \\
\text { heart disease; CVD, cardiovascular disease; CHF, congestive heart failure; NSAIDs, non-steroidal anti- } \\
\text { inflammatory drugs }\end{array}$ & & & & \\
\hline
\end{tabular}

Regarding medication, incident users were more likely to be prescribed hypnotics and analgesics than never users; furthermore, ever users were considerably more likely to be prescribed hypnotics than incident users (Figure 2A). Regarding hospitalization records, ever and incident users were more likely to be hospitalized, especially cancer-related hospitalization and hospitalization due to psychotic disorders (Figure 2B).

\section{Nested case-control study to examine the factors associated with incident antidepressant use}

After excluding ever users of antidepressants from the descriptive cohort, we performed a nested casecontrol study to examine the factors associated with incident antidepressant use. The nested casecontrol design, also known as risk-set sampling, is a type of study design that identifies controls from a group of people who are "at risk" at the index date of the case. ${ }^{24}$ Herein, we defined the day of the first filling of an antidepressant prescription as the index date. For each case, 10 controls were selected, and they were selected from subjects of the same sex and age \pm 3 years; hence, we finally analyzed 14,637 individuals, including 1,330 incident users and 13,307 controls.

The baseline characteristics are compared in Table 2. Incident users had a higher proportion of MetS cases and hypnotic and analgesic users, and they were more likely to experience body weight increase from age 20 and any hospitalization and cancer-related hospitalizations prior to the index date. Table 3 shows the results of both univariable and multivariable conditional logistic regression analyses to examine associations with incident antidepressant use (case). Multivariable model 1 showed significant associations with MetS (odds ratio [OR], 1.30 [95\% confidence interval \{Cl\}: 1.09-1.55]); "quicker" (OR, 0.67 [95\% Cl: 0.57-0.78]) and "late" (OR, 1.23 [95\% Cl: 1.05-1.43]) eating speeds, as compared "normal"; poor sleep (OR, 1.48 [95\% Cl: 1.32-1.67]); "sometimes" (OR, 0.82 [95\% Cl: 0.71-0.94]) and "everyday" (OR, 0.76 [95\% Cl: 0.65-0.90]) drinking habits, as compared "rarely drink"; and cancer-related hospitalizations (OR, 1.76 [95\% Cl: 1.18-2.65] but not with CVD (OR, 1.63 [95\% Cl: 0.89-2.98]). In Model 3, similar results were obtained in addition to significant associations with hypnotics (OR, 1.97 [95\% Cl: 1.47-2.64]), anxiolytics (OR, 3.55 [95\% Cl: $2.79-4.51]$ ), and nonsteroidal anti-inflammatory drugs (NSAIDs) (OR, 1.65 [95\% Cl: 1.18-2.32]). Other metabolic traits, including BMI (OR, 1.03 [95\% Cl: 1.01-1.05]), AC (OR, 1.14 [95\% Cl: 1.07-1.22]), high BP (OR, 1.15 [95\% Cl: 1.00-1.31]), glucose intolerance (OR, 1.24 [95\% Cl: 1.04- 
1.48]), and a >10 kg increase in bodyweight from age 20 (OR, 1.29 [95\% Cl: 1.14-1.46]), were also significantly associated with antidepressant initiation (Table 4). 
Table 2

Baseline characteristics of the risk-set sample

\begin{tabular}{|c|c|c|c|c|c|}
\hline & $\mathbf{N}$ & $\begin{array}{l}\text { Total } \\
N=14,337\end{array}$ & $\begin{array}{l}\text { Incident } \\
\text { users } \\
\mathrm{N}=1,330\end{array}$ & $\begin{array}{l}\text { Controls } \\
N=13,007\end{array}$ & $\begin{array}{l}P \\
\text { value }\end{array}$ \\
\hline Age, years & 14,337 & $46(7)$ & $46(8)$ & $46(7)$ & 0.84 \\
\hline Sex (male) & 14,337 & $9,560(66.7)$ & $886(66.6)$ & $8,674(66.7)$ & 0.96 \\
\hline \multicolumn{6}{|l|}{ Metabolic traits } \\
\hline Metabolic syndrome & 14,337 & $1,689(11.8)$ & $190(14.3)$ & $1,499(11.5)$ & 0.003 \\
\hline Abdominal circumference, $\mathrm{cm}$ & 14,337 & $82(10)$ & $83(10)$ & $82(9)$ & 0.001 \\
\hline $\mathrm{BMI}, \mathrm{kg} / \mathrm{m}^{2}$ & 14,337 & $23.3(3.6)$ & $23.5(3.9)$ & $23.2(3.6)$ & 0.003 \\
\hline SBP, mmHg & 14,337 & $121(16)$ & $121(15)$ & $121(16)$ & 0.57 \\
\hline $\mathrm{DBP}, \mathrm{mmHg}$ & 14,337 & $76(12)$ & $75(11)$ & $76(12)$ & 0.74 \\
\hline Triglyceride, mg/dL & 14,337 & $87(61-131)$ & $91(61-133)$ & $86(61-131)$ & 0.16 \\
\hline HDL cholesterol, mg/dL & 14,337 & $61(51-74)$ & $60(51-71)$ & $61(51-74)$ & 0.012 \\
\hline LDL cholesterol, mg/dL & 14,337 & $\begin{array}{l}121(102- \\
142)\end{array}$ & $\begin{array}{l}122(102- \\
144)\end{array}$ & $\begin{array}{l}121(102- \\
142)\end{array}$ & 0.17 \\
\hline $\mathrm{HbA} 1 \mathrm{c}, \%$ & 11,006 & $5.5(5.3-5.7)$ & $5.5(5.3-5.7)$ & $5.5(5.3-5.7)$ & 0.22 \\
\hline Fasting blood glucose, $\mathrm{mg} / \mathrm{dL}$ & 14,337 & $94(88-101)$ & $95(88-102)$ & $93(88-101)$ & 0.005 \\
\hline \multicolumn{6}{|c|}{ Self-administered lifestyle information } \\
\hline Exercise $>30 \mathrm{~min} /$ day & 13,730 & $2,386(17.4)$ & $208(16.8)$ & $2,178(17.4)$ & 0.56 \\
\hline Physical activity $>60 \mathrm{~min} /$ day & 13,702 & $5,823(42.5)$ & $551(44.5)$ & $5,272(42.3)$ & 0.14 \\
\hline Fast walking speed & 4,685 & $2,181(46.6)$ & $117(39.3)$ & $2,064(47.0)$ & 0.009 \\
\hline Eating speed & 13,710 & & & & $<0.001$ \\
\hline Quicker & & $3,409(24.9)$ & $231(18.6)$ & $3,178(25.5)$ & \\
\hline Normal & & $8,038(58.6)$ & $756(60.9)$ & $7,282(58.4)$ & \\
\hline Late & & $2,263(16.5)$ & $254(20.5)$ & $2,009(16.1)$ & \\
\hline Late supper & 13,702 & $5,407(39.5)$ & $515(41.5)$ & $4,892(39.3)$ & 0.12 \\
\hline \multicolumn{6}{|c|}{$\begin{array}{l}\text { Data are expressed as } \mathrm{N}(\%) \text { for categorical values and mean (standard deviation) for continuous } \\
\text { values. Abbreviations: BMI, body mass index; SBP, systolic blood pressure; DBP, diastolic blood } \\
\text { pressure; HDL, high-density lipoprotein; LDL, low-density lipoprotein; BW, body weight; IHD, ischemic } \\
\text { heart disease; CVD, cardiovascular disease; CHF, congestive heart failure; NSAIDs, non-steroidal anti- } \\
\text { inflammatory drugs }\end{array}$} \\
\hline
\end{tabular}




\begin{tabular}{|c|c|c|c|c|c|}
\hline & $\mathbf{N}$ & $\begin{array}{l}\text { Total } \\
\mathrm{N}=14,337\end{array}$ & $\begin{array}{l}\text { Incident } \\
\text { users } \\
\mathrm{N}=1,330\end{array}$ & $\begin{array}{l}\text { Controls } \\
N=13,007\end{array}$ & $\begin{array}{l}P \\
\text { value }\end{array}$ \\
\hline Skipping breakfast & 13,063 & $2,465(18.9)$ & $223(18.7)$ & 2,242 (18.9) & 0.89 \\
\hline Poor sleep & 13,709 & $4,930(36.0)$ & $552(44.5)$ & $4,378(35.1)$ & $<0.001$ \\
\hline Drinking habits & 14,168 & & & & $<0.001$ \\
\hline Rarely drink & & $6,093(43.0)$ & $632(48.0)$ & $5,461(42.5)$ & \\
\hline Sometimes & & $4,498(31.7)$ & $395(30.0)$ & $4,103(31.9)$ & \\
\hline Everyday & & $3,577(25.2)$ & $291(22.1)$ & $3,286(25.6)$ & \\
\hline Current smoking & 14,315 & $3,806(26.6)$ & $351(26.4)$ & $3,455(26.6)$ & 0.86 \\
\hline $\begin{array}{l}>10 \mathrm{~kg} \text { increase in BW from } \\
\text { age } 20\end{array}$ & 14,337 & $5,619(39.2)$ & $594(44.7)$ & $5,025(38.6)$ & $<0.001$ \\
\hline \multicolumn{6}{|c|}{ Current and past medical history } \\
\hline Hypertension & 14,337 & $2,868(20.0)$ & $273(20.5)$ & $2,595(20.0)$ & 0.62 \\
\hline Diabetes mellitus & 14,337 & $727(5.1)$ & $76(5.7)$ & $651(5.0)$ & 0.26 \\
\hline Dyslipidemia & 14,337 & $5,065(35.3)$ & $506(38.0)$ & $4,559(35.1)$ & 0.030 \\
\hline Self-reported stroke & 14,159 & $56(0.4)$ & $7(0.5)$ & $49(0.4)$ & 0.41 \\
\hline Self-reported IHD & 14,162 & $142(1.0)$ & $12(0.9)$ & $130(1.0)$ & 0.73 \\
\hline \multicolumn{6}{|c|}{ Hospitalization prior to the index date } \\
\hline Any cause & 14,337 & $1,048(7.3)$ & $119(8.9)$ & $929(7.1)$ & 0.016 \\
\hline CVD & 14,337 & $98(0.7)$ & $14(1.1)$ & $84(0.6)$ & 0.086 \\
\hline $\mathrm{CHF}$ & 14,337 & $41(0.3)$ & $6(0.5)$ & $35(0.3)$ & 0.24 \\
\hline Cancer & 14,337 & $203(1.4)$ & $33(2.5)$ & $170(1.3)$ & $<0.001$ \\
\hline \multicolumn{6}{|l|}{ Medication } \\
\hline Hypnotics & 14,337 & $354(2.5)$ & $92(6.9)$ & $262(2.0)$ & $<0.001$ \\
\hline Anxiolytics & 14,337 & $475(3.3)$ & 145 (10.9) & $330(2.5)$ & $<0.001$ \\
\hline Antihypertensive agents & 14,337 & $831(5.8)$ & $113(8.5)$ & $718(5.5)$ & $<0.001$ \\
\hline \multicolumn{6}{|c|}{$\begin{array}{l}\text { Data are expressed as } \mathrm{N}(\%) \text { for categorical values and mean (standard deviation) for continuous } \\
\text { values. Abbreviations: BMI, body mass index; SBP, systolic blood pressure; DBP, diastolic blood } \\
\text { pressure; HDL, high-density lipoprotein; LDL, low-density lipoprotein; BW, body weight; IHD, ischemic } \\
\text { heart disease; CVD, cardiovascular disease; CHF, congestive heart failure; NSAIDs, non-steroidal anti- } \\
\text { inflammatory drugs }\end{array}$} \\
\hline
\end{tabular}




\begin{tabular}{|c|c|c|c|c|c|}
\hline & $\mathbf{N}$ & $\begin{array}{l}\text { Total } \\
\mathrm{N}=14,337\end{array}$ & $\begin{array}{l}\text { Incident } \\
\text { users } \\
\mathrm{N}=1,330\end{array}$ & $\begin{array}{l}\text { Controls } \\
N=13,007\end{array}$ & $\begin{array}{l}P \\
\text { value }\end{array}$ \\
\hline Antidiabetic agents & 14,337 & $224(1.6)$ & $19(1.4)$ & 205 (1.6) & 0.68 \\
\hline NSAIDs & 14,337 & $310(2.2)$ & $50(3.8)$ & $260(2.0)$ & $<0.001$ \\
\hline Statins & 14,337 & $533(3.7)$ & $61(4.6)$ & $472(3.6)$ & 0.079 \\
\hline \multicolumn{6}{|c|}{$\begin{array}{l}\text { Data are expressed as } \mathrm{N}(\%) \text { for categorical values and mean (standard deviation) for continuous } \\
\text { values. Abbreviations: BMI, body mass index; SBP, systolic blood pressure; DBP, diastolic blood } \\
\text { pressure; HDL, high-density lipoprotein; LDL, low-density lipoprotein; BW, body weight; IHD, ischemic } \\
\text { heart disease; CVD, cardiovascular disease; CHF, congestive heart failure; NSAIDs, non-steroidal anti- } \\
\text { inflammatory drugs }\end{array}$} \\
\hline
\end{tabular}


Table 3

Factors associated with the initiation of antidepressants

\begin{tabular}{|c|c|c|c|c|c|}
\hline & \multirow[t]{2}{*}{$\mathbf{N}$} & $\begin{array}{l}\text { Unadjusted } \\
\text { model }\end{array}$ & Model 1 & Model 2 & Model 3 \\
\hline & & OR $(95 \% \mathrm{Cl})$ & OR $(95 \% \mathrm{Cl})$ & OR (95\% Cl) & OR $(95 \% \mathrm{Cl})$ \\
\hline \multicolumn{6}{|l|}{ Metabolic traits } \\
\hline Metabolic syndrome & 14337 & $\begin{array}{l}1.30(1.10- \\
1.53)\end{array}$ & $\begin{array}{l}1.30(1.09- \\
1.55)\end{array}$ & $\begin{array}{l}1.30(1.09- \\
1.55)\end{array}$ & $\begin{array}{l}1.29(1.08- \\
1.54)^{(1.08}\end{array}$ \\
\hline $\begin{array}{l}\text { Abdominal circumference } \\
(/ 10 \mathrm{~cm})\end{array}$ & 14337 & $\begin{array}{l}1.12(1.05- \\
1.19)\end{array}$ & & & \\
\hline BMI & 14337 & $\begin{array}{l}1.03(1.01- \\
1.04)^{-}\end{array}$ & & & \\
\hline $\begin{array}{l}\text { Systolic blood pressure (/10 } \\
\mathrm{mmHg})\end{array}$ & 14337 & $\begin{array}{l}0.99(0.95- \\
1.03)\end{array}$ & & & \\
\hline $\begin{array}{l}\text { Diastolic blood pressure } \\
(/ 10 \mathrm{mmHg})\end{array}$ & 14337 & $\begin{array}{l}1.00(0.94- \\
1.05)\end{array}$ & & & \\
\hline Triglyceride (/10 mg/dL) & 14337 & $\begin{array}{l}1.00(0.99- \\
1.01)\end{array}$ & & & \\
\hline $\begin{array}{l}\text { HDL cholesterol (/10 } \\
\mathrm{mg} / \mathrm{dL})\end{array}$ & 14337 & $\begin{array}{l}0.95(0.91- \\
0.98)\end{array}$ & & & \\
\hline $\begin{array}{l}\text { LDL cholesterol (/10 } \\
\mathrm{mg} / \mathrm{dL})\end{array}$ & 14337 & $\begin{array}{l}1.01(1.00- \\
1.03)\end{array}$ & & & \\
\hline Hba1c & 11006 & $\begin{array}{l}1.11(1.00- \\
1.24)\end{array}$ & & & \\
\hline $\begin{array}{l}\text { Fasting blood glucose (/10 } \\
\mathrm{mg} / \mathrm{dL})\end{array}$ & 14337 & $\begin{array}{l}1.04(1.01- \\
1.07)^{(-01-}\end{array}$ & & & \\
\hline \multicolumn{6}{|c|}{ Self-administered lifestyle information } \\
\hline Exercise $>30 \mathrm{~min} /$ day & 13730 & $\begin{array}{l}0.94(0.81- \\
1.11)\end{array}$ & $\begin{array}{l}1.00(0.85- \\
1.17)\end{array}$ & $\begin{array}{l}1.00(0.86- \\
1.18)\end{array}$ & $\begin{array}{l}1.00(0.86- \\
1.18)\end{array}$ \\
\hline $\begin{array}{l}\text { Physical activity }>60 \\
\mathrm{~min} / \text { day }\end{array}$ & 13702 & $\begin{array}{l}1.09(0.97- \\
1.23)\end{array}$ & & & \\
\hline Fast walking speed & 4685 & $\begin{array}{l}0.69(0.53- \\
0.91)\end{array}$ & & & \\
\hline Eating speed & 12898 & & & & \\
\hline
\end{tabular}

Bolded letters represent $P$ values less than 0.05 . Abbreviations: $\mathrm{OR}$, odds ratio; $\mathrm{Cl}$, confidence interval; $\mathrm{BMI}$, body mass index; SBP, systolic blood pressure; DBP, diastolic blood pressure; HDL, high-density lipoprotein; LDL, low-density lipoprotein; BW, body weight; IHD, ischemic heart disease; CVD, cardiovascular disease; CHF, congestive heart failure; NSAIDs, non-steroidal anti-inflammatory drugs 


\begin{tabular}{|c|c|c|c|c|c|}
\hline & $\mathbf{N}$ & $\begin{array}{l}\text { Unadjusted } \\
\text { model }\end{array}$ & Model 1 & Model 2 & Model 3 \\
\hline & & OR $(95 \% \mathrm{Cl})$ & OR $(95 \% \mathrm{Cl})$ & OR $(95 \% \mathrm{Cl})$ & OR (95\% Cl) \\
\hline Quicker & & $\begin{array}{l}0.67(0.57- \\
0.79)\end{array}$ & $\begin{array}{l}0.67(0.57- \\
0.78)\end{array}$ & $\begin{array}{l}0.69(0.59- \\
0.81)\end{array}$ & $\begin{array}{l}0.69(0.59- \\
0.81)\end{array}$ \\
\hline Normal & & Reference & Reference & Reference & Reference \\
\hline Late & & $\begin{array}{l}1.22(1.05- \\
1.42)\end{array}$ & $\begin{array}{l}1.23(1.05- \\
1.43)^{(1.05}\end{array}$ & $\begin{array}{l}1.25(1.07- \\
1.46)\end{array}$ & $\begin{array}{l}1.25(1.07- \\
1.46)\end{array}$ \\
\hline Late supper & 13702 & $\begin{array}{l}0.94(0.81- \\
1.11)\end{array}$ & & & \\
\hline Skipping breakfast & 13063 & $\begin{array}{l}0.97(0.83- \\
1.14)\end{array}$ & & & \\
\hline Poor sleep & 13709 & $\begin{array}{l}1.48(1.31- \\
1.67)\end{array}$ & $\begin{array}{l}1.48(1.32- \\
1.67)\end{array}$ & $\begin{array}{l}1.41(1.25- \\
1.59)\end{array}$ & $\begin{array}{l}1.41(1.25- \\
1.60)\end{array}$ \\
\hline Drinking habits & 14168 & & & & \\
\hline Rarely drink & & Reference & Reference & Reference & Reference \\
\hline Sometimes & & $\begin{array}{l}0.91(0.80- \\
1.03)\end{array}$ & $\begin{array}{l}0.82(0.71- \\
0.94)\end{array}$ & $\begin{array}{l}0.84(0.73- \\
0.98)\end{array}$ & $\begin{array}{l}0.85(0.73- \\
0.98)\end{array}$ \\
\hline Everyday & & $\begin{array}{l}0.81(0.70- \\
0.94)\end{array}$ & $\begin{array}{l}0.76(0.65- \\
0.90)\end{array}$ & $\begin{array}{l}0.78(0.66- \\
0.92)\end{array}$ & $\begin{array}{l}0.78(0.66- \\
0.92)\end{array}$ \\
\hline Current smoking & 14315 & $\begin{array}{l}0.99(0.86- \\
1.13)\end{array}$ & & & \\
\hline $\begin{array}{l}>10 \mathrm{~kg} \text { increase in BW from } \\
\text { age } 20\end{array}$ & 14337 & $\begin{array}{l}1.30(1.15- \\
1.46)\end{array}$ & & & \\
\hline \multicolumn{6}{|c|}{ Current and past medical history } \\
\hline Hypertension & 14337 & $\begin{array}{l}1.03(0.89- \\
1.19)\end{array}$ & & & \\
\hline Diabetes mellitus & 14337 & $\begin{array}{l}1.16(0.90- \\
1.48)\end{array}$ & & & \\
\hline Dyslipidemia & 14337 & $\begin{array}{l}1.14(1.01- \\
1.29)\end{array}$ & & & \\
\hline Self-reported stroke & 14159 & $\begin{array}{l}1.39(0.62- \\
3.07)\end{array}$ & & & \\
\hline
\end{tabular}

Bolded letters represent $P$ values less than 0.05 . Abbreviations: $\mathrm{OR}$, odds ratio; $\mathrm{Cl}$, confidence interval; $\mathrm{BMI}$, body mass index; SBP, systolic blood pressure; DBP, diastolic blood pressure; HDL, high-density lipoprotein; LDL, low-density lipoprotein; BW, body weight; IHD, ischemic heart disease; CVD, cardiovascular disease; CHF, congestive heart failure; NSAIDs, non-steroidal anti-inflammatory drugs 


\begin{tabular}{|c|c|c|c|c|c|}
\hline & \multirow[t]{2}{*}{$\mathbf{N}$} & $\begin{array}{l}\text { Unadjusted } \\
\text { model }\end{array}$ & Model 1 & Model 2 & Model 3 \\
\hline & & OR $(95 \% \mathrm{Cl})$ & OR (95\% Cl) & OR (95\% Cl) & OR (95\% Cl) \\
\hline Self-reported IHD & 14162 & $\begin{array}{l}0.91(0.50- \\
1.65)\end{array}$ & & & \\
\hline Hospitalization pr & ex date & & & & \\
\hline Any cause & 14337 & $\begin{array}{l}1.29 \\
1.58)\end{array}$ & & & \\
\hline CVD & 14337 & $\begin{array}{l}1.66(0.94- \\
2.95)\end{array}$ & $\begin{array}{l}1.63(0.89- \\
2.98)\end{array}$ & & $\begin{array}{l}1.75(0.96- \\
3.22)\end{array}$ \\
\hline CHF & 14337 & $\begin{array}{l}1.70(0.71- \\
4.07)\end{array}$ & & & \\
\hline Cancer & 14337 & $\begin{array}{l}1.98(1.35- \\
2.89)\end{array}$ & $\begin{array}{l}1.76(1.18- \\
2.65)\end{array}$ & & $\begin{array}{l}1.45(0.95- \\
2.22)\end{array}$ \\
\hline Medication & & & & & \\
\hline Hypnotics & 14337 & $\begin{array}{l}3.59(2.80- \\
4.59)\end{array}$ & & $\begin{array}{l}1.95(1.25- \\
1.59)\end{array}$ & $\begin{array}{l}1.97(1.47- \\
2.64)\end{array}$ \\
\hline Anxiolytics & 14337 & $\begin{array}{l}4.76(3.86- \\
5.86)\end{array}$ & & $\begin{array}{l}3.61(2.84- \\
4.58)\end{array}$ & $\begin{array}{l}3.55(2.79- \\
4.51)\end{array}$ \\
\hline NSAIDs & 14337 & $\begin{array}{l}1.92(1.41- \\
2.61)\end{array}$ & & $\begin{array}{l}1.67(1.19- \\
2.33)\end{array}$ & $\begin{array}{l}1.65(1.18- \\
2.32)\end{array}$ \\
\hline $\begin{array}{l}\text { Bolded letters repr } \\
\text { BMl, body mass ir } \\
\text { lipoprotein; LDL, I } \\
\text { cardiovascular dis }\end{array}$ & $\begin{array}{l}\text { less th } \\
\text { olic blo } \\
\text { protein } \\
\text { ngestive }\end{array}$ & $\begin{array}{l}0.05 . \text { Abbre } \\
\text { pressure; DB } \\
\text { W, body weig } \\
\text { eart failure; } N\end{array}$ & $\begin{array}{l}\text { ions: OR, odd } \\
\text { liastolic blooc } \\
\text { IHD, ischemic } \\
\text { IDs, non-sterc }\end{array}$ & $\begin{array}{l}\text { ratio; } \mathrm{Cl} \text {, confic } \\
\text { rressure; } \mathrm{HDL} \\
\text { eart disease; } \\
\text { lal anti-inflamr }\end{array}$ & $\begin{array}{l}\text { nce interval; } \\
\text { igh-density } \\
\text { ID, } \\
\text { atory drugs }\end{array}$ \\
\hline
\end{tabular}


Table 4

Associations of metabolic traits and BW increase history with incident use of antidepressants

\begin{tabular}{|c|c|c|c|c|c|c|}
\hline \multirow[t]{2}{*}{ Metabolic traits } & \multicolumn{2}{|l|}{ Model 1} & \multicolumn{2}{|l|}{ Model 2} & \multicolumn{2}{|l|}{ Model 3} \\
\hline & OR $(95 \% \mathrm{Cl})$ & $\begin{array}{l}P \\
\text { value }\end{array}$ & $\begin{array}{l}\text { OR }(95 \% \\
\mathrm{Cl})\end{array}$ & $\begin{array}{l}P \\
\text { value }\end{array}$ & $\begin{array}{l}\text { OR }(95 \% \\
\mathrm{Cl})\end{array}$ & $\begin{array}{l}P \\
\text { value }\end{array}$ \\
\hline Metabolic syndrome & $\begin{array}{l}1.30(1.09- \\
1.55)\end{array}$ & 0.003 & $\begin{array}{l}1.30(1.09- \\
1.55)\end{array}$ & 0.004 & $\begin{array}{l}1.29 \\
(1.08- \\
1.54)\end{array}$ & 0.005 \\
\hline BMI & $\begin{array}{l}1.03(1.01- \\
1.04)\end{array}$ & 0.001 & $\begin{array}{l}1.03(1.01- \\
1.05)\end{array}$ & 0.001 & $\begin{array}{l}1.03 \\
(1.01- \\
1.05)\end{array}$ & 0.001 \\
\hline $\begin{array}{l}\text { Abdominal circumference } \\
(/ 10 \mathrm{~cm})\end{array}$ & $\begin{array}{l}1.14(1.07- \\
1.22)\end{array}$ & $<0.001$ & $\begin{array}{l}1.15(1.07- \\
1.22)\end{array}$ & $<0.001$ & $\begin{array}{l}1.14 \\
(1.07- \\
1.22)\end{array}$ & $<0.001$ \\
\hline $\begin{array}{l}>10 \mathrm{~kg} \text { increase in BW } \\
\text { from age } 20\end{array}$ & $\begin{array}{l}1.31(1.16- \\
1.48)\end{array}$ & $<0.001$ & $\begin{array}{l}1.30(1.15- \\
1.47)\end{array}$ & $<0.001$ & $\begin{array}{l}1.29 \\
(1.14- \\
1.46)\end{array}$ & $<0.001$ \\
\hline High blood pressure & $\begin{array}{l}1.13(0.99- \\
1.28)\end{array}$ & 0.070 & $\begin{array}{l}1.16(1.01- \\
1.32)\end{array}$ & 0.030 & $\begin{array}{l}1.15 \\
(1.00- \\
1.31)\end{array}$ & 0.042 \\
\hline Glucose intolerance & $\begin{array}{l}1.24(1.04- \\
1.48)\end{array}$ & 0.015 & $\begin{array}{l}1.24(1.04- \\
1.48)\end{array}$ & 0.017 & $\begin{array}{l}1.24 \\
(1.04- \\
1.48)\end{array}$ & 0.017 \\
\hline Dyslipidemia & $\begin{array}{l}1.11(0.96- \\
1.27)\end{array}$ & 0.16 & $\begin{array}{l}1.07(0.93- \\
1.24)\end{array}$ & 0.33 & $\begin{array}{l}1.07 \\
(0.93- \\
1.24)\end{array}$ & 0.35 \\
\hline $\begin{array}{l}\text { Number of metabolic } \\
\text { components }\end{array}$ & $\begin{array}{l}1.11(1.03- \\
1.20)\end{array}$ & 0.005 & $\begin{array}{l}1.11(1.03- \\
1.20)\end{array}$ & 0.006 & $\begin{array}{l}1.11 \\
(1.03- \\
1.19)\end{array}$ & 0.008 \\
\hline \multicolumn{7}{|c|}{$\begin{array}{l}\text { Model } 1 \text { was adjusted for regular exercise habits }>30 \text { minutes a day, poor sleep, drinking habits, } \\
\text { hospitalization due to or associated with CVD, and cancer. Model } 2 \text { was adjusted for regular exercise } \\
\text { habits }>30 \text { minutes a day, poor sleep, drinking habits, use of hypnotics, anxiolytics, and NSAIDs. } \\
\text { Model } 3 \text { was adjusted for Model } 1 \text { plus use of hypnotics, anxiolytics, and NSAIDs. }\end{array}$} \\
\hline
\end{tabular}

\section{Discussion}

This study clearly demonstrated the relative associations of various medical and lifestyle factors with the incident use of antidepressants using large-scale real-world data from health insurance claims and health checkups in Japan. The major finding of the present study was that MetS; poor sleep; and the use of hypnotics, anxiolytics, and NSAIDs were associated with antidepressant initiation. It is noteworthy that while hospitalization was not significantly associated with the outcome, MetS and other metabolic 
abnormalities, which are pre-symptomatic conditions, were significantly associated with antidepressant initiation.

We also demonstrated a dose-response association between the number of metabolic components and incident antidepressant use. In particular, we found a significant association of obesity, high BP, and glucose intolerance with depression. The pathways from MetS to depression could be biological or social ${ }^{25}$, a phenomenon that can be explained by the physiological consequences of obesity, including higher inflammation ${ }^{26,27}$ and the psychological/social consequences of MetS or obesity. However, most of the health-related behaviors assessed by the questionnaire, such as exercise habits, late supper, skipping breakfast, and smoking, were not significantly different between the cases and controls. Notably, information on physical activity or exercise habits was collected using self-administered questionnaires rather than quantitative methods. More detailed research is required to investigate physical activity and exercise habits more quantitatively and accurately through a quantitative physical activity using biometric sensors and detailed questionnaires on exercise habits.

Regarding psychosocial factors, physical and mental stress can be common causes of MetS and depression. The study subjects were corporate insurance beneficiaries comprising corporate employees and their dependents, and mental stress is potentially caused by the work itself, human relationships in the workplace, and family issues in this population. Physical stress may be caused by shift work. These not only trigger depression, but can also result in obesity and MetS through overeating, sleep disorders, and effects on various metabolic systems. However, this study did not include information such as work shifts and work-related stress, and further studies are required to identify modifiable factors and identify solutions to overcome both health issues.

We also produced interesting results regarding lifestyle information. First, we found that alcohol consumption is potentially protective against depression. Several previous studies have shown consistent results regarding the protective effect of alcohol against depression. ${ }^{28-32}$ Although our results did not show a clear benefit of daily physical activity, modifiable lifestyle factors including sleep and exercise habits, are potential candidates for intervention in both conditions, that is, depression and MetS. Daily exercise habits are particularly suitable lifestyle interventions, as a large body of evidence demonstrates the effectiveness of exercise on depression and sleep disorders. ${ }^{33-36}$

Regarding prescription medicine, the use of hypnotics, anxiolytics, and NSAIDs at baseline was associated with incident antidepressant use. Studies have shown that insomnia is associated with depression and anxiety disorders. ${ }^{37,38}$ However, depression is often underdiagnosed in primary-care settings or for older patients ${ }^{39}$, and such medications might have been prescribed as supportive care for patients with symptoms and complaints related to depression but not formally diagnosed. Hence, individuals with underdiagnosed and undertreated depression may be hidden among those who have been prescribed these drugs. 
Finally, a history of hospitalization for malignant diseases was also associated with incident antidepressant use without adjusting for medication. However, the association was attenuated after adjusting for medication information. This may be due to the mediation effects of anxiety and insomnia on the association. Previous studies have demonstrated that anxiety, depression, and insomnia are associated with cancer as well as with increased adverse outcomes, including mortality and psychosocial problems in cancer survivors. ${ }^{40-43}$ The present study reaffirms the importance of psychological care for those who experience cancer-related hospitalization.

This study had certain limitations. First, the present study was observational and did not demonstrate any causality. However, our results revealed various medical and lifestyle factors associated with depression, which we believe provide valuable insights into workplace mental and physical health. Second, we classified depression based on specific, prescribed classes of antidepressants, that is, selective serotonin reuptake inhibitors (SSRIs), serotonin-noradrenalin reuptake inhibitors (SNRIs), or noradrenergic and specific serotonin antidepressants (NaSSAs), thus potentially leading to misclassification bias since those who were treated with other classes of medication or those who had not been treated with medication were not diagnosed with depression. This type of misclassification is unavoidable in large-scale database studies. However, the main purpose of this study was to explore and generate hypotheses for future research. Therefore, sufficient findings have been generated from this analysis. Third, there was a concern regarding selection bias. Those who were taking sick leave due to depression might not have undergone health checkups in the workplace. However, in our country, the number of individuals who undergo health checkups is high in the working generations. Finally, most of the lifestyle information was derived from self-administered questionnaires, thus potentially undermining accuracy and objectivity. We may consider using activity monitoring, such as gyro meters, to gain objective personal activity information in future studies.

In conclusion, metabolic abnormalities; poor sleep habits; cancer-related hospitalization; and the use of hypnotics, anxiolytics, and NSAIDs were associated with incident antidepressant use in working-age individuals. Lifestyle intervention could be the subsequent step in reducing both mental and physical burdens among individuals in their prime.

\section{Methods}

\section{Data source and ethical issues}

The data source comprised anonymized, processed receipt information and medical checkup data provided by health insurance associations in Japan contracted with PREVENT Co., Ltd, and are stored at the company. The present study analyzed data from the administrative claims-based database that included information on 134,677 individuals who underwent health examinations at least once and were under health insurance coverage between April, 2014 and March, 2019, targeted at corporate employees and their dependents in Japan. All data was extracted and processed on November 17, 2020. We conducted this study in accordance with the guidelines of the Declaration of Helsinki. The institutional 
ethics committee of Nagoya University Graduate School of Medicine formally approved this study (Approval No.2020-0142). The Ethical Review Committee for Observational Research is a officially approved and registered organization (No. 15000226). Since the study data were provided anonymously, and the study participants did not receive any intervention, informed consent for study participation was waived.

\section{Definitions}

We obtained the following information from checkup data: sex, age, BMI, BP, and AC data; laboratory data on HbA1c, fasting blood glucose, TG, HDL-c, and LDL-c; smoking and alcohol habits; and lifestyle and behavior, including weight changes, exercise habits, physical activity, walking speed, eating speed and habits, and poor sleep. Exercise habits were defined as exercising to sweat lightly for $>30$ min per session, twice weekly, for over a year. Physical activity was defined as walking or performing an equivalent amount of physical activity for $>1 \mathrm{~h}$ per day. Walking speed was categorized into the following three categories: "quicker" than others, "normal", and "late". Late supper was defined as eating supper $2 \mathrm{~h}$ before bedtime more than three times a week. Skipping breakfast was defined as skipping breakfast more than three times a week. Bodyweight change was defined as a $>10 \mathrm{~kg}$ increase in body weight from the age of 20 years.

We defined the following medicines or their combination as antidepressants: SSRI, SNRI, and NaSSA. We did not include other types of classical antidepressants, including tricyclic or tetracyclic antidepressants, or serotonin antagonists and reuptake inhibitors, since they have been used for purposes other than depression treatment. We defined "ever" users as individuals whose last antidepressant prescription filling was earlier than 30 days after the baseline date and "never" users as those who had never been prescribed an antidepressant within 30 days after the baseline date (Figure 3). "Incident" users were defined as individuals who initiated antidepressants $>30$ days after the baseline date.

Additionally, we obtained baseline data on medication history other than antidepressants, disease name, hospitalization, and procedure from the claims data prior to 30 days after the baseline. A positive medication history was defined as at least one prescription filling during the study period. Data on antihypertensive agents, antidiabetic agents, statins, hypnotics, and NSAIDs were collected based on WHO-ATC codes (Supplementary Table 1). Detailed information on drug code combinations representing these drugs is available on GitHub. (https://github.com/PREVENT-

Inc/MyscopeMasterList/tree/master/nagoya-u/medical_conditions_and_depression).

Hospitalization information was also obtained from claims data prior to 30 days after the baseline date. Hospitalizations due to specific causes were defined using a combination of the International Statistical Classification of Diseases and Related Health Problems (ICD)-10 codes in the hospitalization information of medical claims data. Herein, we classified hospitalizations into the following categories: any cause, CVD, congestive heart failure, cancer, and psychotic disorders. CVD-related hospitalization was defined as having any of the following diseases: acute myocardial infarction, congestive heart failure, and cerebrovascular disease. The combinations of ICD-10 codes have been described in a previous study by 
Quan et al. ${ }^{44}$ Hospitalizations due to mental disorders were classified based on the ICD-10 classification of mental and behavioral disorders. ${ }^{45}$

\section{Statistical analysis}

For between-group comparisons, categorical variables are expressed as numbers and percentages, and continuous variables as the median (interquartile range) or mean (standard deviation).

In the nested case-control study, a between-group comparison was performed in a fashion similar to the comparison of the overall study participants. Univariable and multivariable conditional logistic regression was used to examine the factors associated with the incident prescription of antidepressants. Covariates included age; sex; smoking and drinking habits; physical activity; poor sleep; the use of hypnotics, anxiolytics, and NSAIDs; and hospitalizations due to CVD and cancer. Hospitalization records were utilized in a time-updated manner, implying that hospitalization records were collected not only from baseline but also from data prior to the index dates. Two-sided statistical significance was set at $P<0.05$. All analyses were performed using Stata version 17.0 software (StataCorp, TX, USA).

\section{Declarations}

\section{Acknowledgments}

The authors deeply acknowledge all the participants of the present study. The authors also acknowledge Editage for providing editorial and publication support.

\section{Author contributions}

TI analyzed the data and wrote the paper; TT and DS prepared the data; MM, YH, YY, MA, and SM interpreted the results.

\section{Availability Statement}

The data used in this study is the property of PREVENT Co., Ltd. and the purpose of use is restricted by PREVENT's policy on data utilization. The data used in this study was also used under contract and is not available to the public. Inquiries about the data can be made via GoogleForm [https://forms.gle/iWbhLjEx157dkXZB9], and can available after a license agreement is signed.

\section{Competing Interests Statement}

The authors declare no competing interests.

\section{Funding}

This research did not receive any specific grant from funding agencies in the public, commercial, or notfor-profit sectors. 


\section{References}

1. James, S. L. et al. Global, regional, and national incidence, prevalence, and years lived with disability for 354 diseases and injuries for 195 countries and territories, 1990-2017: a systematic analysis for the Global Burden of Disease Study 2017. Lancet392, 1789-1858 (2018).

2. Meng, R. et al. Association of Depression with All-Cause and Cardiovascular Disease Mortality among Adults in China. JAMA Netw. Open3, (2020).

3. Gilman, S. E. et al. Depression and mortality in a longitudinal study: 1952-2011. CMAJ189, E1304E1310 (2017).

4. Carney, R. M. \& Freedland, K. E. Depression, mortality, and medical morbidity in patients with coronary heart disease. Biological Psychiatry vol. 54 241-247 (2003).

5 . Katon, W. J. et al. The association of comorbid depression with mortality in patients with type 2 diabetes. Diabetes Care28, 2668-2672 (2005).

6. Nouwen, A. et al. Longitudinal associations between depression and diabetes complications: a systematic review and meta-analysis. Diabetic Medicine vol. 36 1562-1572 (2019).

7. Mezuk, B., Eaton, W. W., Albrecht, S. \& Golden, S. H. Depression and type 2 diabetes over the lifespan: A meta-analysis. Diabetes Care31, 2383-2390 (2008).

8. Musselman, D. L., Evans, D. L. \& Nemeroff, C. B. The relationship of depression to cardiovascular disease: Epidemiology, biology, and treatment. Archives of General Psychiatry vol. 55 580-592 (1998).

9. Hare, D. L., Toukhsati, S. R., Johansson, P. \& Jaarsma, T. Depression and cardiovascular disease: A clinical review. European Heart Journal vol. 35 1365-1372 (2014).

10. Zhang, Y., Chen, Y. \& Ma, L. Depression and cardiovascular disease in elderly: Current understanding. Journal of Clinical Neuroscience vol. 47 1-5 (2018).

11. Organisation for Economic Co-operation and Development. Sickness, Disability and Work: Breaking the Barriers. OECD https://www.oecd.org/publications/sickness-disability-and-work-breaking-thebarriers-9789264088856-en.htm (2010) doi:10.1787/9789264088856-EN.

12. Savikko, A., Alexanderson, K. \& Hensing, G. Do mental health problems increase sickness absence due to other due to other diseases? Soc. Psychiatry Psychiatr. Epidemiol.36, 310-316 (2001).

13. Kessler, R. C., Ormel, J., Demler, O. \& Stang, P. E. Comorbid Mental Disorders Account for the Role Impairment of Commonly Occurring Chronic Physical Disorders: Results from the National Comorbidity Survey. J. Occup. Environ. Med.45, 1257-1266 (2003).

14. Stewart, W. F., Ricci, J. A., Chee, E., Hahn, S. R. \& Morganstein, D. Cost of Lost Productive Work Time among US Workers with Depression. J. Am. Med. Assoc.289, 3135-3144 (2003).

15. Kessler, R. C. The costs of depression. Psychiatric Clinics of North America vol. 35 1-14 (2012).

16. Robinson, R. G. Poststroke depression: Prevalence, diagnosis, treatment, and disease progression. Biological Psychiatry vol. 54 376-387 (2003). 
17. Krebber, A. M. H. et al. Prevalence of depression in cancer patients: A meta-analysis of diagnostic interviews and self-report instruments. Psycho-Oncology vol. 23 121-130 (2014).

18. Campayo, A., Gómez-Biel, C. H. \& Lobo, A. Diabetes and depression. Curr. Psychiatry Rep.13, 26-30 (2011).

19. Marazziti, D., Rutigliano, G., Baroni, S., Landi, P. \& Dell'Osso, L. Metabolic syndrome and major depression. CNS Spectrums vol. 19 293-304 (2014).

20. Pan, A. et al. Bidirectional association between depression and metabolic syndrome: A systematic review and meta-analysis of epidemiological studies. Diabetes Care35, 1171-1180 (2012).

21. Eckel, R. H., Grundy, S. M. \& Zimmet, P. Z. The metabolic syndrome. in Lancet vol. 365 1415-1428 (Lancet, 2005).

22. Grundy, S. M. et al. Diagnosis and management of the metabolic syndrome: An American Heart Association/National Heart, Lung, and Blood Institute scientific statement. Circulation vol. 112 2735-2752 (2005).

23. Takahara, M. \& Shimomura, I. Metabolic syndrome and lifestyle modification. Reviews in Endocrine and Metabolic Disorders vol. 15 317-327 (2014).

24. Langholz, B. \& Goldstein, L. Risk set sampling in epidemiologic cohort studies. Stat. Sci.11, 35-53 (1996).

25. Casanova, F. et al. Higher adiposity and mental health: causal inference using Mendelian randomization. Hum. Mol. Genet.30, 2371-2382 (2021).

26. Miller, A. H. \& Raison, C. L. The role of inflammation in depression: From evolutionary imperative to modern treatment target. Nature Reviews Immunology vol. 16 22-34 (2016).

27. Lawlor, D. A., Smith, G. D. \& Ebrahim, S. Association of insulin resistance with depression: Cross sectional findings from the British women's heart and health study. Br. Med. J.327, 1383-1384 (2003).

28. García-Esquinas, E. et al. Moderate alcohol drinking is not associated with risk of depression in older adults. Sci. Rep.8, (2018).

29. Cheng, H. G., Chen, S., McBride, O. \& Phillips, M. R. Prospective relationship of depressive symptoms, drinking, and tobacco smoking among middle-aged and elderly community-dwelling adults: Results from the China Health and Retirement Longitudinal Study (CHARLS). J. Affect. Disord.195, 136-143 (2016).

30. Paulson, D. et al. The relationship between moderate alcohol consumption, depressive symptomatology, and C-reactive protein: the Health and Retirement Study. Int. J. Geriatr. Psychiatry33, 316-324 (2018).

31. Gea, A. et al. Alcohol intake, wine consumption and the development of depression: The PREDIMED study. BMC Med.11, (2013).

32. Bellos, S. et al. Longitudinal association between different levels of alcohol consumption and a new onset of depression and generalized anxiety disorder: Results from an international study in primary 
care. Psychiatry Res.243, 30-34 (2016).

33. Schuch, F. B. \& Stubbs, B. The Role of Exercise in Preventing and Treating Depression. Curr. Sports Med. Rep.18, 299-304 (2019).

34. Sarris, J., O’Neil, A., Coulson, C. E., Schweitzer, I. \& Berk, M. Lifestyle medicine for depression. BMC Psychiatry vol. 14 (2014).

35. Lopresti, A. L., Hood, S. D. \& Drummond, P. D. A review of lifestyle factors that contribute to important pathways associated with major depression: Diet, sleep and exercise. Journal of Affective Disorders vol. 148 12-27 (2013).

36. Reid, K. J. et al. Aerobic exercise improves self-reported sleep and quality of life in older adults with insomnia. Sleep Med.11, 934-940 (2010).

37. Chang, P. P., Ford, D. E., Mead, L. A., Cooper-Patrick, L. \& Klag, M. J. Insomnia in young men and subsequent depression: The Johns Hopkins Precursors Study. Am. J. Epidemiol.146, 105-114 (1997).

38. Neckelmann, D., Mykletun, A. \& Dahl, A. A. Chronic insomnia as a risk factor for developing anxiety and depression. Sleep30, 873-880 (2007).

39. Allan, C. E., Valkanova, V. \& Ebmeier, K. P. Depression in older people is underdiagnosed. in Practitioner vol. 258 19-22 (Practitioner, 2014).

40. Walker, J. et al. Major Depression and Survival in People with Cancer. Psychosom. Med.83, 410-416 (2021).

41. Hong, J. S. \& Tian, J. Prevalence of anxiety and depression and their risk factors in Chinese cancer patients. Support. Care Cancer22, 453-459 (2014).

42. Irwin, M. R. Depression and insomnia in cancer: Prevalence, risk factors, and effects on cancer outcomes. Curr. Psychiatry Rep.15, (2013).

43. Savard, J. \& Morin, C. M. Insomnia in the context of cancer: A review of a neglected problem. Journal of Clinical Oncology vol. 19 895-908 (2001).

44. Quan, H. et al. Coding algorithms for defining comorbidities in ICD-9-CM and ICD-10 administrative data. Med. Care43, 1130-1139 (2005).

45. World Health Organization. The ICD-10 classification of mental and behavioural disorders: Clinical descriptions and diagnostic guidelines. (1992).

\section{Figures}




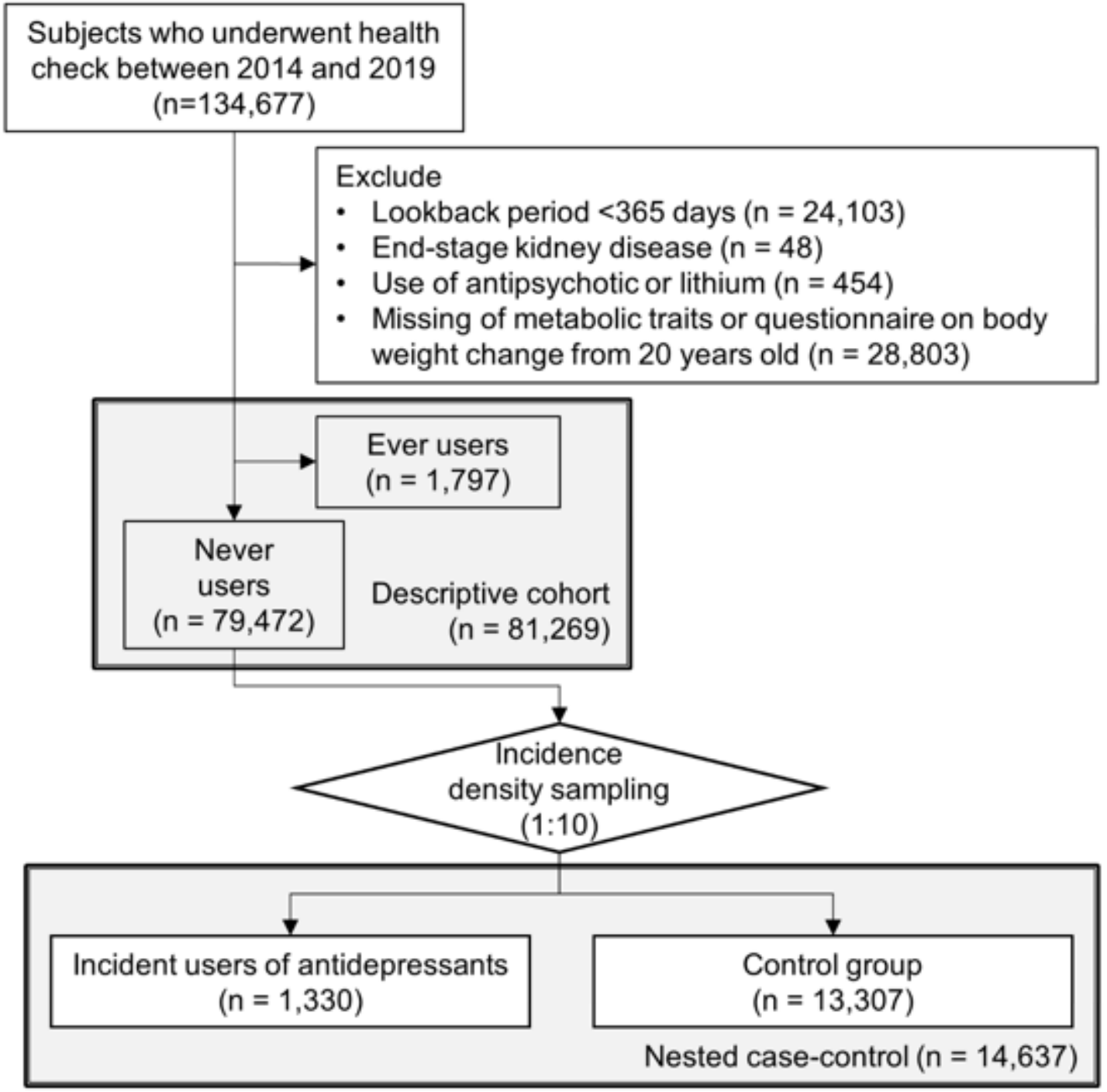

\section{Figure 1}

Flow diagram of study subject selection 

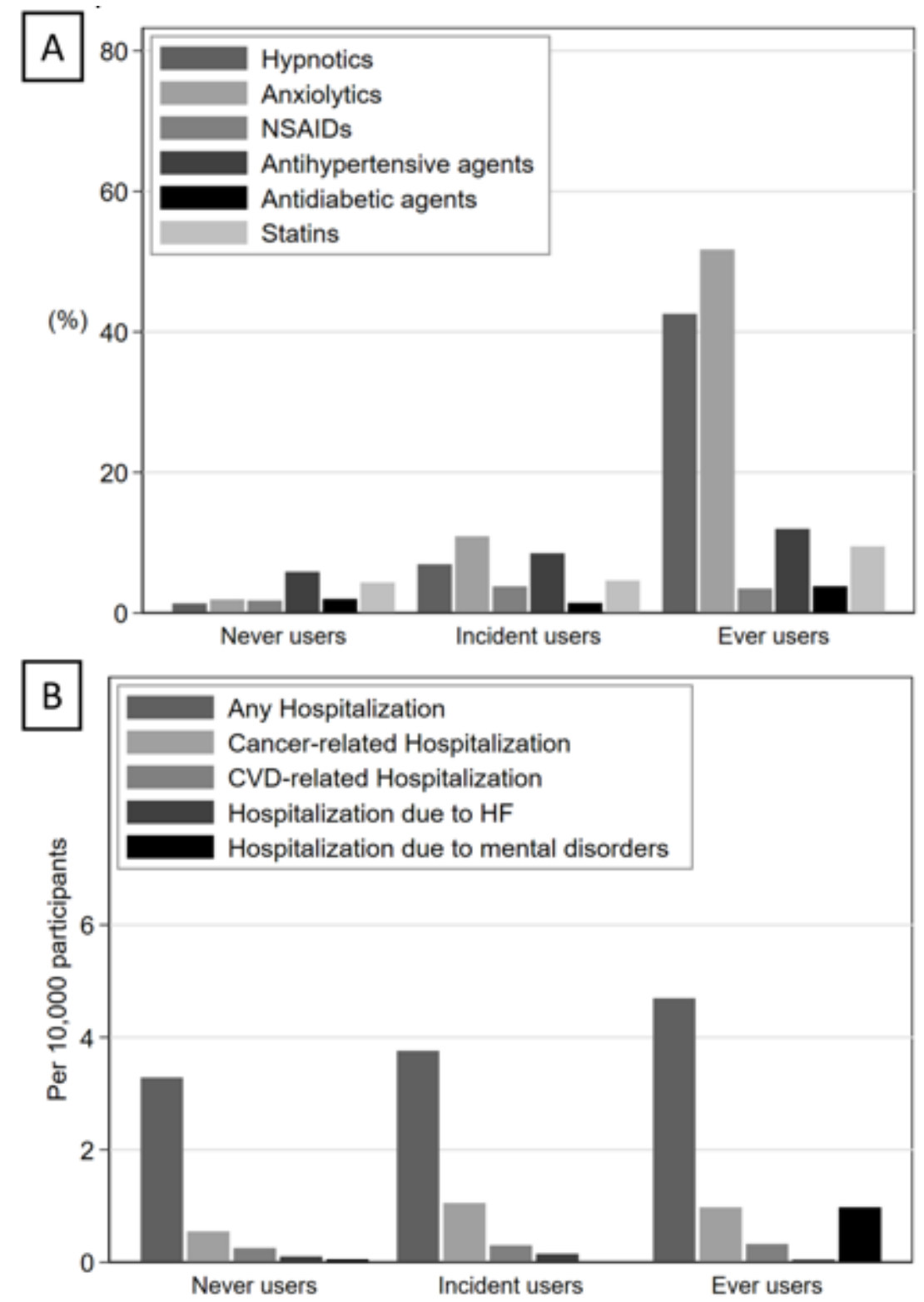

Figure 2

Medication and hospitalization of never, ever, and incident users of antidepressants 


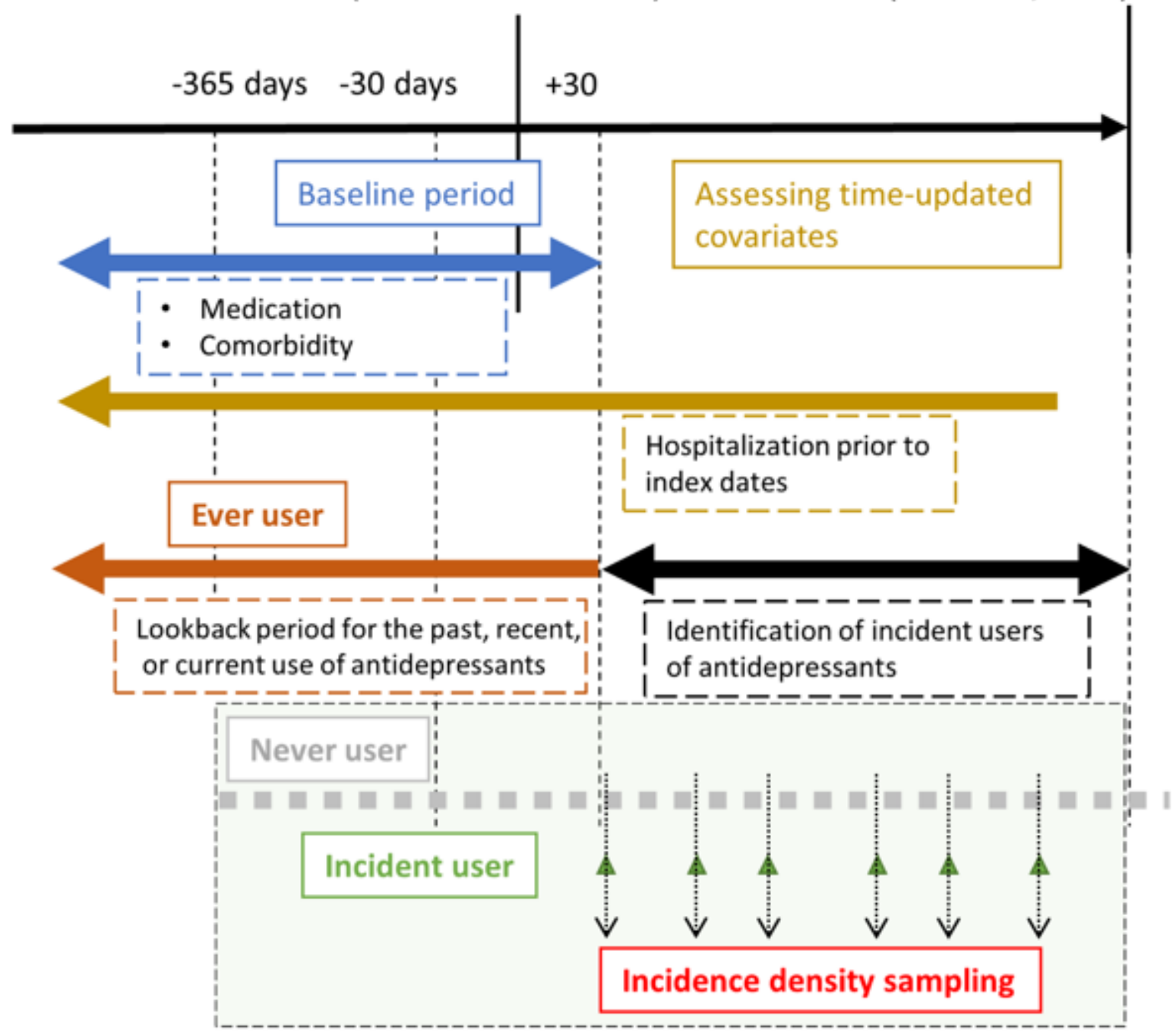

Figure 3

Design diagram of the study

\section{Supplementary Files}

This is a list of supplementary files associated with this preprint. Click to download.

- SupplementaryTable1.docx 